\title{
Numerical Investigation of Unsteady Flowfield of DN25 Flow Meter with Pressure Chamber
}

\author{
A. Gedikli ${ }^{1}$, H. Krisch ${ }^{1}$, E. von Lavante ${ }^{2}$ \\ ${ }^{1}$ Krohne Messtechnik GmbH, Duisburg, Germany \\ 2 University of Duisburg-Essen, Faculty of Engineering
}

\begin{abstract}
:
In the present investigation, a new design flow meter with pressure detection has been optimized and investigated numerically. In the classical design of the KROHNE flow meter a paddle is used as a sensor, containing piezoelectric elements. When flow passes around a bluff body periodic flow separation develops which is responsible for the oscillation of the paddle. Thus the piezoelectric elements transform the mechanical movement of the paddle in electrical signal which can be evaluated in signal processing. Such a sensor construction is restricted to medium temperatures until $240^{\circ} \mathrm{C}$. In order to achieve an accurate measurement at higher temperatures, for example at $500^{\circ} \mathrm{C}$, a new design flow meter has been developed. Instead of using a paddle which is positioned to detect periodic flow fluctuations, the periodic change of pressure is detected through two openings which are positioned at the side wall close to the bluff body and leading to a pressure chamber. The periodically changing pressure acts on a membrane and thus with a suitable sensor design it is possible to detect the underlying frequency.
\end{abstract}

Key words: Flow Simulation, High Temperature Measurement, Flow Meter

\section{Introduction}

In the chemical industry and many other industrial processes the measurement of massand volumetric flow rate is essential. In order to maintain correct flow rate in a pipe system the accurate data from a flow meter is necessary. One simple flow measurement device applicable here is the so-called vortex-shedding flow meter (VSFM). The vortex flow meter consists of a bluff body installed in a measurement section. When a fluid flows over the bluff body a von Karman vortex street occurs. The appearance of a periodic vortex shedding downstream of the bluff body is a natural phenomenon which exists at certain Reynolds numbers. A quasi linearity between vortex shedding frequency and bulk velocity exists in a certain Reynolds number range. In that operation range the dimensionless Strouhal number is predominantly independent of the Reynolds number and remains approximately constant. This is the reason for the usage of a certain bluff body with a sharp leading edge in order to generate a well defined separation. By determining the vortex shedding frequency downstream of the bluff body i.e. with a paddle as detector the volumetric flow rate can be obtained directly providing a representing reference value of the K-Factor. This information is implemented into the DSP electronics resulting in flow rate specification. The K-Factor designates the ratio between vortex shedding frequency and the volumetric flow rate. In a certain Reynolds number range the K-Factor remains approximately constant, leading to a constant Strouhal number. The reference K-Factor designates an average value of the K-Factors at different Reynolds numbers and thus represents the whole operational range of the vortex flow meter. The K-Factor depends upon the bluff body shape and the size of the tube in which the bluff body is attached. The K-Factor and the Strouhal number do not remain exactly constant and undergo small changes depending on the flow conditions.

Nowadays, commercial vortex flow meters use a large variety of bluff body shapes, test sections (conical inflow and outflow, constrictors of various shapes) and signal detection systems (pick-up). The corresponding unsteady flow fields have been studied by, among others, Johnson [4], Fureby [1], Madabhushi et. al. [5] and Gedikli [12] using mostly numerical simulations. The signal detection and processing have been discussed by Hans et. al. [2] and [3]. The potential for 
improvement of signal quality by modifying the shape of the bluff body was investigated by von Lavante et al. [6]). It has been also observed that a slight uncontrolled modification of the assumed geometry of a particular VSFM, e.g. shape, location relative to the surrounding casing and change of shape due to wear caused by particles suspended in the metered fluid, could cause a shift of its characteristic frequencies, leading to unreliable volumetric flow data. The influence of the manufacturing tolerances on the accuracy of VSFMs and abrasion by particles suspended in the metered fluid has been investigated in [7] and [8] by von Lavante et al. . A detailed study of the flow field in small size commercial VSFMs with inflow and outflow conditioned by a Venturi nozzle and a diffusor has been published by von Lavante et al. in [9]. Finally, the effects of upstream disturbances on the accuracy of VSFMs have been studied by von Lavante et al. [10, 11]. Gedikli [12] followed a similar approach to study flow disturbances introduced upstream of the flow meter by different installations and parameters using numerical simulation techniques.

In the present investigation, a DN 25 VSFM has been numerically investigated whereby a pressure chamber acted as a vortex shedding frequency detector. The considered working medium was air. As the velocity was periodically changing at a considered location downstream of the bluff body the pressure was changing in the same manner due to the coupling between the velocity and the pressure fields. The pressure waves propagated through the side wall openings into the pressure chamber, making the detection of the time wise change of the pressure possible. Three simulations at different Reynolds numbers (corresponding to $u_{m}=20 \mathrm{~m} / \mathrm{s}, 40 \mathrm{~m} / \mathrm{s}$ and $70 \mathrm{~m} / \mathrm{s}$ ) were carried out assuming high temperature conditions, $\mathrm{T}=773 \mathrm{~K}\left(500^{\circ} \mathrm{C}\right)$ at atmospheric pressure as well as one simulation at high temperature $(\mathrm{T}=773 \mathrm{~K})$ and high pressure ( $p=350$ bar) at $u_{m}=70 \mathrm{~m} / \mathrm{s}$. At high temperature some flow parameters changed, including the dynamic viscosity and thermal conductivity making manual adjustments in the software program necessary. Accordingly, the Reynolds number was changing as well. It was interesting to observe the linearity of the relationship between the frequency and the bulk velocity or the constancy of the K-Factor in the DN 25 vortex flow meter under these extreme conditions. However, for the analysis it is useful to consider a wider range of Reynolds numbers in order to get wide range Strouhal number distribution. In the present case, the flow in the
DN25 VSFM was characterized by a certain blocking ratio, given by the length of the bluff body characterized by $d / l=0,67$. The height of the bluff body $\mathrm{d}$ was the most important geometric parameter affecting the vortex shedding frequency. The unsteady computations were carried out using a commercial, three-dimensional, unsteady and compressible solver of the Navier-Stokes equations provided by STARCCM+. As the temperature in the flow field was high a stronger coupling between the continuity and the momentum equations and the energy equation had to be taken into account. The consideration of the coupling becomes more and more important for large thermal conductivity and Mach-Number. In order to account for this effect the coupled solver was used in each case. The simulations at $p=1$ bar were carried out using the ideal gas law to account for the density changes. In the simulation at high pressure and temperature the real gas effects were considered using the Redlich Kwong equation as equation of state. The next figure shows a simplified cross sectional cut through the bluff body and the pressure chamber. As can be seen from figure 1 above, no geometrical modifications were considered at the end sides of the bluff body. According to [12, 17], geometrical modifications at the end sides of the bluff body can modulate the vortex shedding frequency.

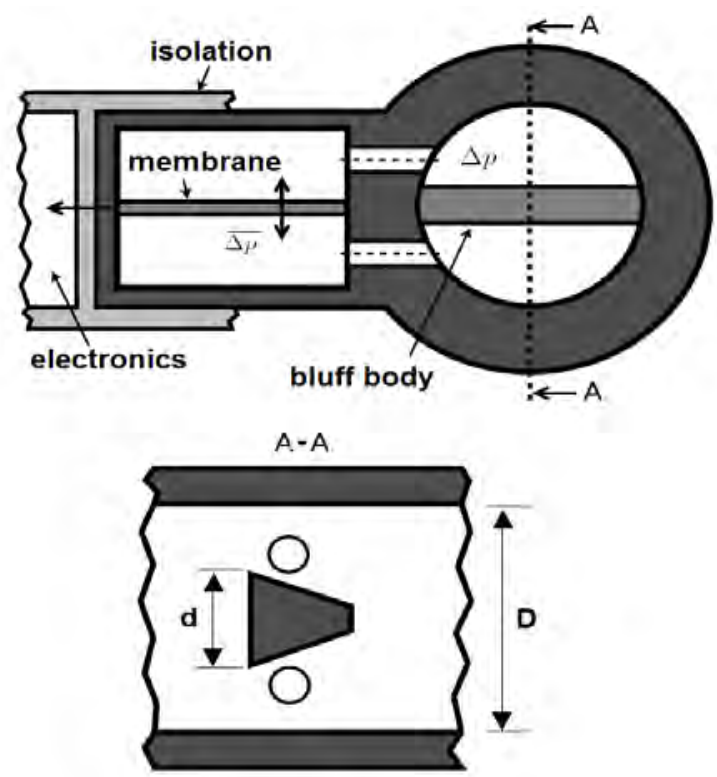

Fig. 1. Cross section through investigated vortex flow meter with pressure chamber

In the present paper a theoretical treatment of the flow in a vortex flow meter is offered, revealing some interesting flow behavior under these extreme flow conditions. In order to create a reference basis some numerical 
results were used for comparison at higher Reynolds numbers which were obtained under normal conditions and at different bulk velocities [12]. In the past many simulations were done under normal conditions which give as result good agreement to experimental results by using certain models. The mentioned reference simulations at standard conditions were done under these models [12]. Some details are offered in the "Numerical Investigation" part.

\section{Numerical Investigation}

Based upon a 3-D CAD model a mesh was constructed with the software program GRIDGEN. Figure 2 offers one view of the constructed mesh. It is a hybrid mesh which consists of structured and unstructured cell regions. The mesh consists of 660000 cells.

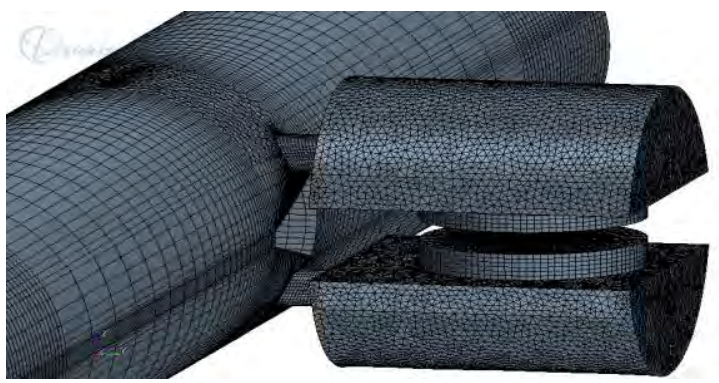

Fig. 2. View of the constructed 3-D hybrid mesh

In the mesh construction an inlet and outlet length of approx. $L=3 D$ was considered as well. In the numerical simulations fully developed turbulent velocity profiles at the considered bulk velocities were imported at the flow domain inlet. Thus the simulations were done under non-disturbed conditions. As the considered Reynolds numbers based on the pipe diameter $D$ were larger than the critical value of 2300 the flow in the pipe tube was assumed to be turbulent. A turbulent flow is characterized by the dominantly stochastic movement of smaller and larger vortices at very small time and space scales. As the constructed mesh cannot resolve the small turbulent structures the turbulent flow fields were calculated using the unsteady RANS equations whereby the effect of turbulence was considered by modeling the turbulent stress tensor by a turbulent model. Thus it was possible to account for the turbulent effects without resolving the complex flow field. The Realizable k-epsilon turbulence model was used in each case as turbulence model. This model is appropriate for turbulent flows with local transitional effects and gives good agreement between numerical and experimental results $[12,13]$. The bluff body height was so small (corresponding Reynolds number based upon $d$ is small) that the flow around the bluff body is laminar. Therefore, laminar vortices as primary vortices were periodically shedding at the leading edge of the bluff body and were convected downstream of the bluff body. At high temperature and low pressure the flow behaved like an ideal gas so that in this case the ideal gas law could be used as the equation of state. In the unsteady simulation at high temperature and high pressure, $p=350$ bar, the density was calculated by considering real gas effects. In order to investigate the thermal influence on the vortex shedding frequency the coupled solver was used. As mentioned before the thermal properties have been specified in the simulation program manually according to the considered temperature and pressure. If the temperature is increasing the dynamic viscosity is increasing as well. The increasing behavior is typical for gases. For liquids the opposite behavior is true. In Figure 3 a diagram is depicted which shows the dynamic viscosity versus temperature behavior.

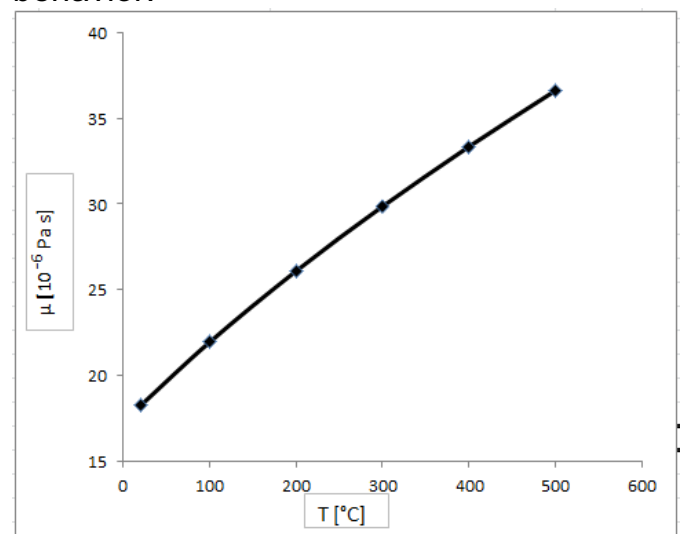

Fig. 3. Dynamic viscosity versus temperature for air

In order to have a reference basis with regard to the numerical Strouhal numbers obtained presently under the mentioned extreme flow conditions, the results of some simulations at different Reynolds numbers under atmospheric pressure $p=1$ bar and at $T=300 \mathrm{~K}$ were used from [12]. The models used for these simulations were identified by some validations in [12]. The simulations were done at higher Reynolds numbers in order to obtain a whole Strouhal number distribution to estimate the reliability of the obtained numerical results at higher temperatures. The investigated vortex flow meter configuration under standard conditions employed as the reference basis was of the same configuration consisting of a bluff body of a similar shape (with no geometrical modifications at the bluff body end sides) and the same height $d$. The only 
difference was in the bluff body length and the detector type. As detector for determining the time wise lift coefficient a paddle was used. The vortex shedding frequencies were evaluated through the lift force acting on the paddle.

\section{Numerical Results}

The instantaneous velocity and Mach number distributions in the flow field at $u_{m}=70 \mathrm{~m} / \mathrm{s}$ are depicted in Figure 4. The local velocity reaches $180 \mathrm{~m} / \mathrm{s}$ in the bluff body region due to the blockage effect. The Mach number distribution at $u_{m}=70 \mathrm{~m} / \mathrm{s}$ shows that local compressible effects occur as the maximum Mach number is around 0,33 .

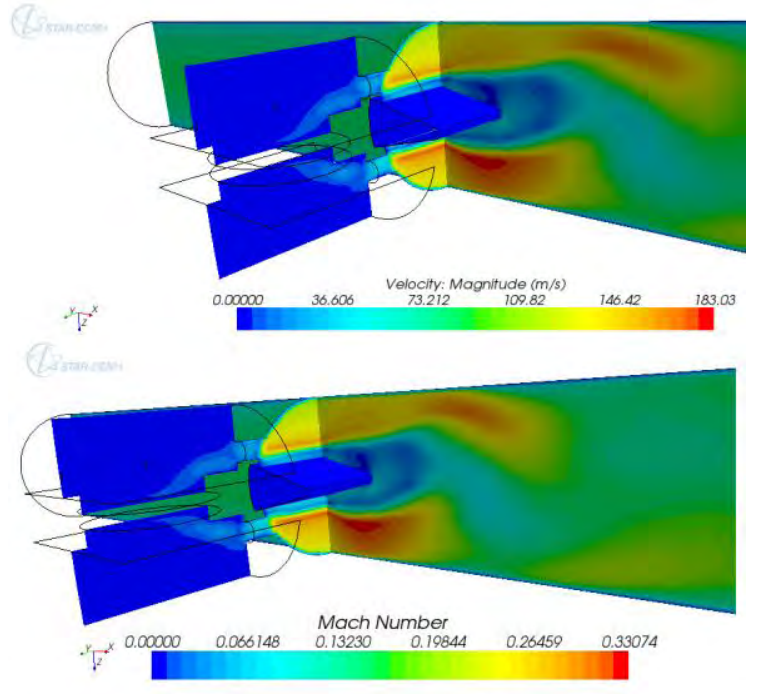

Fig. 4. Velocity and Mach number distribution

However, closer examination of the Mach number distribution in the pressure chamber reveals that the Mach number tends in this region to zero. As the Mach number is an indicator for the compressibility of the flow it can be concluded that the flow field is of hybrid character. The flow in the main pipe is locally compressible, whereby the flow in the pressure chamber behaves incompressibly as the Mach number approaches zero. The flow in the pressure chamber has an elliptic flow character which means that from theoretical point of view the pressure waves propagate with very high speed. Thus the periodic time wise change of pressure at the bluff body is transferred into the pressure chamber according to linear wave propagation and acts as a periodic force on the membrane surfaces. Comparing with the maximum local Mach number observed at room temperature $\left(\mathrm{M}_{\max }=0,54\right.$ at $\left.\mathrm{u}_{\mathrm{m}}=70 \mathrm{~m} / \mathrm{s}\right)$ the Mach number in the present investigation at $70 \mathrm{~m} / \mathrm{s}$ is due to the higher temperature lower as the speed of sound is larger. Accordingly, the high temperature tends to reduce the compressible effects. This has an effect on the Strouhal number because the Strouhal number depends, among other parameters, also on the Mach number. From Schlichting [14] it is known that increasing Mach number causes higher values of drag force coefficient. This leads to the statement that increasing temperature causes a decrease in inertia forces which leads to lower values of Strouhal number.

It is interesting to consider lower vortex shedding frequencies as compared with the frequencies occurring at room temperature and at atmospheric pressure. Accordingly, the numerical reference K-Factor has a value of approximately 74968 . In the next figure, the KFactor distribution obtained as a function of the bulk velocity is plotted whereby the dashed line indicates the reference K-Factor at standard conditions having a value of about 85000 .

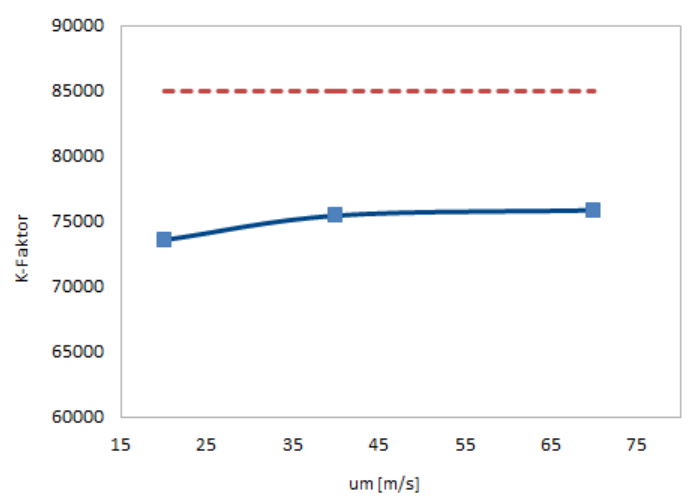

Fig. 5. K-Factor distribution as a function of bulk velocity compared with the reference case (dashed line)

In this plot one can observe that the linearity is not affected in the Reynolds number range considered. According to Fiedler [15] the Strouhal number is widely independent of pressure and dynamic viscosity which means that the constancy of this parameter is not affected (theoretically). Only the absolute value of this parameter has decreased to a lower value.

Figure 6 shows the Strouhal number versus Reynolds number plot which was obtained numerically. The blue curve denotes the Strouhal number distribution obtained under standard conditions in a similar configuration (idealized VSFM configuration with no geometrical modifications at the bluff body end sides as the investigated configuration here) at different bulk velocities, with a paddle as detector. The dashed line gives a linear interpolation through these points. As this 
interpolating line passes almost horizontally and captures a wider Reynolds number range it is possible to compare the numerical results at lower Reynolds numbers obtained under high temperature and $p=1$ bar (green points) with the standard condition. The shift from the dashed line is clearly visible. Considering the dashed line shows that at lower Reynolds numbers a decrease of Strouhal number can be expected.

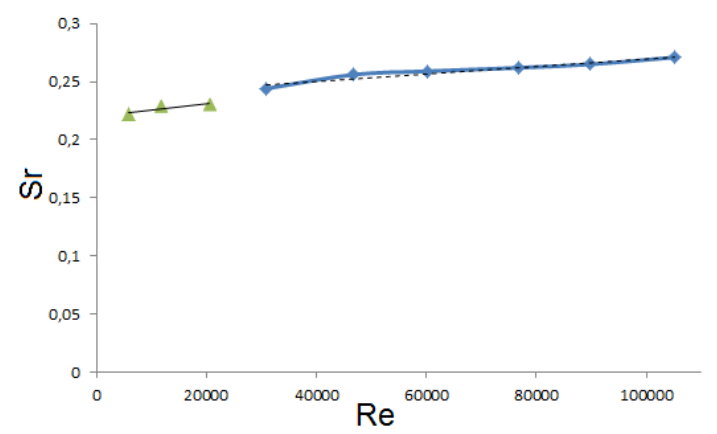

Fig. 6 Strouhal-Reynolds number plot of the numerical results obtained under standard conditions (blue curve) and at high temperature (green points) at $p=1 \mathrm{bar}$

Further discussion is concerned with decrease of Strouhal number as compared with the dashed line at lower Reynolds numbers.

Thus following question arises from these observations: What is the reason for the further decrease in vortex shedding frequency and thus in Strouhal number at higher temperatures under atmospheric pressure?

As the vortex shedding frequency was smaller than in the reference case, the dimensionless Strouhal number was lower as well. The main reason for the further decrease in vortex shedding frequency at lower Reynolds numbers can be sought in the fact that the dynamic viscosity was higher than in the reference case. The dynamic viscosity is a strong function of temperature. The density of the fluid is lower as well. Therefore, the effective Reynolds number which is based on the height of the bluff body becomes smaller and thus according to [16] the Strouhal number becomes smaller as well. The authors of [16] state a universal formula which shows the dependency of Strouhal number on the Reynolds number based on bluff body height. According to [16] this equation can be written as follows:

$$
S r=0,266-\frac{1,0610}{\sqrt{\operatorname{Re}_{d}}}
$$

But it is expected that more parameters play an important role at these extreme flow conditions.
Due to the fact that the flow parameters in the flow field are coupled with each other, the influence of temperature, specific heat, thermal conductivity and the decreasing Mach number at higher temperatures plays an important role. Therefore, the Strouhal number is a complex function of Prandtl number, Eckert number, Temperature ratio, Mach number,... .

Interestingly, at high pressure $p=350$ bar the vortex shedding frequency decreases even further from $2402 \mathrm{~Hz}$ to $2362,4 \mathrm{~Hz}$. In the next table the flow data obtained presently at $\mathrm{u}_{\mathrm{m}}=70 \mathrm{~m} / \mathrm{s}$ are compared:

Tab. 1: Comparison of Flow Data at $70 \mathrm{~m} / \mathrm{s}$

\begin{tabular}{|c|c|c|c|}
\hline $\begin{array}{c}\mathrm{T} / \\
{ }^{\circ} \mathrm{C}\end{array}$ & $\mathrm{P} / \mathrm{bar}$ & $\mathrm{Red}$ & $\mathrm{Sr}$ \\
\hline 500 & 1 & 25135 & 0,2305 \\
\hline 500 & 350 & 5918815 & 0,2260 \\
\hline
\end{tabular}

Due to the high pressure the density in the flow domain is 300 times larger so that the Reynolds number becomes approx. 235 times larger (small increase in dynamic viscosity is considered). But it is important to note that increasing of Reynolds number by increasing the pressure at a considered bulk velocity is not causing an excessively higher unsteadiness in the flow field. Therefore, it is consistent to obtain frequencies which are in the same order of magnitude. According to many 2-D simulations on a simplified configuration it becomes clear that an increase of pressure at a considered fix bulk velocity tends to reduce the vortex shedding frequency by a small amount. As can be seen from table 1 the Reynolds numbers differ by a significant value. The Reynolds number is a dimensionless parameter which indicates the ratio of inertia forces to viscous forces. The flow field under 350bar is highly turbulent. Thus the turbulent viscosity ratio which was modeled in the computations induced a large amount of inertia into the flow field. The mentioned pressure effect exists in compressible flows where the dynamics of the vortex shedding phenomenon are modified due to the higher inertia induced in the flow domain. The next plot shows both lift coefficients obtained at $u_{m}=70 \mathrm{~m} / \mathrm{s}$ on the bluff body surface. It can be seen that the dynamics of the flow field at high pressure is changed due to the excessively high pressure. A phase shift of the signals can be seen. Thus the vortex shedding frequency was affected. It can be concluded that for compressible flows the Strouhal number is also weakly dependend on pressure. 
However, the pressure is modulating the vortex shedding frequency by a small amount so that one could use a reference Strouhal number (an average value over the considered Reynolds number range) which represents the whole Reynolds number range so that the required accuracy can be maintained. In case of water it is suggested that no pressure effect such here exists due to the incompressibility of the medium.

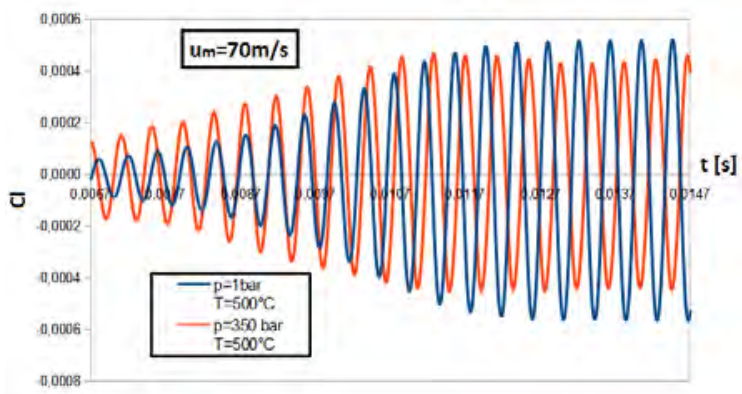

Fig. 7. Comparison of the lift-coefficients

\section{Conclusions}

The present paper deals with the numerical treatment of a flow in a DN 25 vortex flow meter with pressure chamber under high temperature conditions $\left(T=773 \mathrm{~K}\right.$ at $u_{m}=20 \mathrm{~m} / \mathrm{s}, 40 \mathrm{~m} / \mathrm{s}$ and $70 \mathrm{~m} / \mathrm{s})$ as well as high pressure $(p=350$ bar at $\mathrm{u}_{\mathrm{m}}=70 \mathrm{~m} / \mathrm{s}$ ). As reference basis some validated numerical results were used for comparison at

\section{References}

[1] C. Fureby, Large-eddy simulation of turbulent anisochoric flows, AIAA Journal, Vol. 33, No. 7, pp. 1263-1272, 1995.

[2] Hans, V., Poppen, G., Lavante, E. v., Perpeet, S., Interaction between vortices and ultrasonic waves in vortex-shedding flow meters, FLUCOME '97, Hayama, 1997.

[3] Hans, V., Poppen, G., Lavante, E. v., Perpeet, S., Vortex-shedding flow meters and ultrasound detection: signal processing and bluff body geometry, Flow Measurement and Instrumentation 9, pp. 7982, 1998.

[4] Johnson, M.W., Computation of flow in a vortexshedding flow meter, Flow Measurement and Instrumentation, pp. 201-208, 1990 .

[5] Madabhushi, R.K., Choi, D., Barber, T.J., Unsteady simulations of turbulent flow behind a triangular bluff body, AIAA Paper, pp. 97-3182, 1997. higher Reynolds numbers. The present study allowed the estimation of the Strouhal number distribution over a wider Reynolds number range. The obtained vortex shedding frequencies and thus the Strouhal numbers were approximately $11 \%$ lower than in the reference case. The reason for this decrease in vortex shedding frequencies lies in the fact that due to the high temperature the effective Reynolds number based on the bluff body height becomes lower, thus reducing the Strouhal number. In the case of high pressure the opposite behavior was expected as the Reynolds number becomes very large due to the high density. But according to the numerical results the obtained vortex shedding frequency is lower than at $p=1$ bar. The reason for this lies in the fact that the unsteadiness of the flow field is widely independent of pressure and density. The frequency of the unsteady movement is widely depending on the bulk velocity and bluff body height. The further decrease of vortex shedding frequency of around $1,6 \%$ comes from the high inertia induced into the flow field. The inertial force is proportional to the density of the fluid. Thus this is reducing by a small amount the unsteadiness because the von Karman vortex street has changed his structure downstream of the bluff body under the high pressure.

[6] von Lavante, E., Yao, J. and Perpeet, S., Effects of Disturbed Inflow on Vortex-Shedding from a Bluff Body, AIAA paper 2000-2220, 2000.

[7] von Lavante, E., Nath, B. and Färber, J., Effects of Manufacturing Tolerances on the Accuracy of Vortex-Shedding Flow Meters, XVII IMEKO World Congress on Metrology, Dubrovnik, 2003.

[8] E. von Lavante, U. Banaszak, M. G. Yilmaz, O. Ricken and R. Höcker, Effects of Shape Change due to Wear on the Accuracy of Vortex-Shedding Flow Meters, Proceedings of the 14th Int. Conf. on Flow Measurement Flomeko 2007, Johannesburg, RSA, September 2007.

[9] E. von Lavante, U. Banaszak, T. Jubin, S. Tournillon, H. Krisch, Numerical and Experimental Investigation of Small Size Vortex-Shedding Flow Meter, Proceedings of the 7th Int. Symposium "Metrologia 2008", Havana, Cuba, May 2008.

[10] von Lavante, E., Cambier, P., Vandermarliere, S., Banaszak, U., Krisch, H. and Tournillon, Numerical and Experimental Study of Effects of Upstream Disturbance on Accuracy of VortexShedding Flow Meter, Proceedings of the XIX 
IMEKO World Congress on Metrology, Lisbon, September 2009.

[11] von Lavante, E., Gedikli, A., Thibaut, A., Tournillon, S. and Krisch, H., Effects of Upstream Butterfly Valve on the Accuracy of a Vortex Flow Meter, Proceedings of the 15th Flow Measurement Conference

[12] Gedikli A. O., Numerische und experimentelle Untersuchung der instationären Strömungsfelder von Wirbelzählerkonfigurationen, Dissertation, 2012.

[13] Ansys Userguide, Basics to Turbulence Modelling

[14] Hermann Schlichting, Boundary Layer Theory, McGraw Hill, 1951

[15] O. Fiedler, Strömungs- und Durchflussmesstechnik, Oldenborg, 1992.

[16] T. Vit, M. Ren, Z. Travnicek, F. Marsik, C.C.M. Rindt, The influence of temperature gradient on Strouhal-Reynolds number relationship for water and air, Experimental Thermal and Fluid Science 31, 751760, 2007

[17] Ali-Osman Gedikli, E. von Lavante, Sylvain Tournillon, Henrik Krisch, Installation considerations of bluff bodies in vortex flow meters, Metrology, 2011. 